\title{
Estudio sincrónico de obsolescencia de la literatura: el caso de la Ley de Lotka
}

\author{
Rubén Urbizagástegui Alvarado *
}

Artículo recibido: 19 de agosto de 2013.

Artículo aceptado: 19 de noviembre de 2013.

\section{Resumen}

Se analiza la obsolescencia de la literatura producida sobre la Ley de Lotka hasta 2010. En ese periodo no parece haber un patrón de obsolescencia definido. Sin embargo, los datos acumulados muestran una comportamiento obsolescente con una tasa de decrecimiento anual del 3.9 \% y una tasa de duplicación cada 17.4 años. La estadística indica un buen ajuste al modelo exponencial, con un $\mathrm{R}^{2}$ de 0.985 para la literatura a un nivel de significancia de 0.01 .

Palabras clave: Obsolescencia de la literatura; Ley de Lotka; Productividad de autores; Cienciometría; Bibliometría; Informetría.

* Universidad de California, Riverside, CA, USA. ruben@ucr.edu

INVESTIGACIÓN BiBLIOTECOLÓGICA, Vol.28, Núm. 63, mayo/agosto, 2014, México, ISSN: 0187-358X. pp. 85-113 


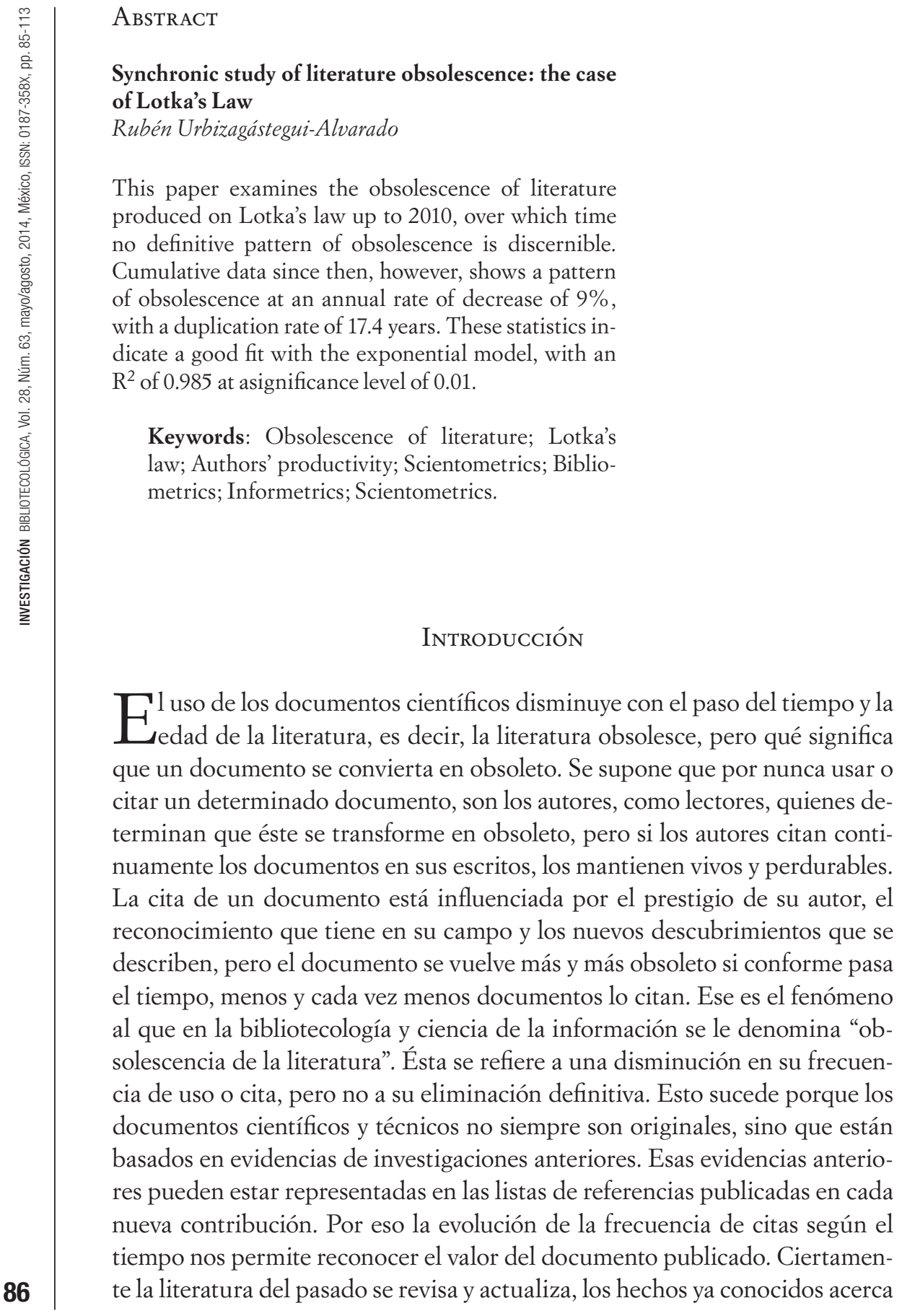


de un fenómeno se incorporan y se fusionan con los nuevos conocimientos acerca de ese fenómeno. Por lo tanto esos hechos son reescritos y reinterpretados en términos de nuevas teorías como correcciones y refinamientos de los artículos publicados que fueron puestos en circulación por las revistas científicas, pero no se sabe cuándo esa literatura se transforma en "obsoleta". Los estudios sobre la obsolescencia de las publicaciones se han convertido en comunes, alentados por el trabajo de Price (1965: 512), quien sugirió que "cada año aproximadamente el $10 \%$ de todos los artículos 'mueren' al no volver a ser citados nuevamente". Los datos recogidos para medir el envejecimiento de los documentos han dado lugar a la conclusión general de que el uso de la literatura declina con los años, de acuerdo con una distribución exponencial negativa, aunque otros autores postulan una distribución lognormal como la más adecuada para medir la obsolescencia de la literatura (Egghe \& Ravichandra Rao, 1992b; Gupta, 1998).

Existen dos métodos de estudio de la obsolescencia: el método sincrónico y el método diacrónico. La obsolescencia sincrónica estudia el uso en el pasado de una muestra de documentos en los cuales la vida media sería la mediana estadística (el punto en el cual se agrupan el $50 \%$ de las frecuencias de las citas) contando los años en orden cronológico inverso. La obsolescencia diacrónica necesita de la selección de un punto en el pasado para dar un vistazo hacia el futuro. Sin embargo, algunos autores argumentan que los estudios sincrónicos y diacrónicos producen los mismos resultados (Stinson, 1981; Stinson \& Lancaster, 1987), sugiriendo preferencia por el método sincrónico. Ambos estudios pueden hacerse también retrospectivamente (Egghe \& Rousseau, 2000).

Si bien es posible saber cuál es la vida media de la literatura, aún no podemos saber cuándo realmente comienza a envejecer y deja de ser citada, ni las razones por las cuales ésta deja de ser citada. ¿Es el acceso al documento lo que influye en la obsolescencia de la literatura? ¿El idioma en que está escrito el documento influye en esta obsolescencia? ¿O es el descubrimiento de nuevos modelos o métodos de medición o evaluación de la disciplina lo que influye?

No siempre es posible hacer el análisis de la obsolescencia usando los índices de citas impresos ni los que están disponibles en línea como el Science Citation Index, el Social Science Citation Index, etc., en primer lugar porque estos índices de citas favorecen la producción anglo-sajona en el proceso de recopilación de los documentos, en menoscabo de la producción de otras regiones; en segundo lugar porque también hacen hincapié en la colección de documentos en el idioma inglés, una vez más a expensas de otros idiomas. Por estas razones la única opción para llevar a cabo esta investigación fue 
desarrollar una base de datos propia para después examinar, analizar, evaluar y comunicar los resultados.

Por las razones apuntadas el objetivo de este trabajo es analizar la existencia o inexistencia de la "obsolescencia de la literatura" en una muestra de documentos publicados del 2007 al 2009 (86 documentos citantes), dedicados a estudiar la Ley de Lotka, frente a un total de 663 documentos citables y disponibles publicados desde 1926 hasta 2006. El modelo teórico está explicado en capítulo aparte. Incidentalmente se estudiarán los idiomas de los documentos incluidos en la muestra y los idiomas más frecuentemente citados por los documentos publicados en inglés, portugués y español de la muestra de 86 documentos. Se usará la ley de Bradford para identificar a los autores citados en el núcleo y la zona de frontera; es decir, a aquellos más frecuentemente citados y cuyos artículos tendrán mayores posibilidades de escapar de la "obsolescencia".

En general este tipo de estudios han sido llevados a cabo contabilizando todas las citas incluidas en una muestra de documentos publicados durante un periodo específico sin ningún tipo de separación y/o discriminación. En este caso, hay interés en la obsolescencia de los documentos dedicados a analizar la Ley de Lotka, que también citarán otros documentos anteriores que igualmente estudiaron y/o aplicaron la Ley de Lotka.

\section{MARCo teórico}

El término "obsolescencia" apareció por primera vez en la obra de Gosnell (1943). Más tarde, durante la realización de la Conferencia Internacional sobre Información Científica celebrada en Washington, se afirmó que:

[...] muchos estudios han demostrado que la vida útil de un ítem de información científica es diferente en los distintos campos de la ciencia. La verdadera vida-media de un ítem particular de información puede ser definida como el tiempo, después de su publicación, que se compone de la mitad de los usos (referencias) o búsquedas de este ítem de información. Por supuesto esto es extremadamente difícil de evaluar; sin embargo, vale la pena hacerlo. En vez de eso, estamos obligados a usar lo que podría llamarse la vida media retrospectiva de un ítem de información similar, por ejemplo, artículos en una revista. Esto puede ser definido como el tiempo contado retroactivamente a partir de una fecha determinada, en la cual hayan ocurrido la mitad de las solicitudes de información o referencias. Este periodo es de 2 años para la física y de 15 años para la biología (Bernal, 1958: 86).

Siguiendo esta misma idea de vida media, Burton \& Kebler (1960) pos- 
vida media significa "la mitad de la vida activa" o el tiempo durante el cual fue publicada la mitad de la literatura. Las investigaciones parecían indicar resultados diferentes, tanto así que Bourne (1965) señaló que la mayoría de las "vidas medias" reportadas eran válidas solamente para las muestras estudiadas, y que no podían ser generalizadas para todas las actividades científicas o técnicas. Poco después, Ewing (1966) llevó a cabo un estudio diacrónico de los artículos publicados en una revista de física y encontró que el número de citas disminuía a medida que crecía el año de las publicaciones. Esta tasa de envejecimiento era más dramática cuando se realizaban ajustes en función del crecimiento de la literatura publicada en esa revista. La "intensidad de las citas" fue siete veces mayor en 1955 que en 1964, y mostró una vida media de 3.5 años y una tasa de obsolescencia de 8.0 años.

El interés por comprender la obsolescencia de la literatura continuó creciendo y Line (1970a) trató de precisar el significado del término "vida media”, ya que en su opinión el sentido comúnmente usado era inadecuado y de un valor limitado. Si la tasa de crecimiento de las colecciones es más rápida o más lenta que la tasa de crecimiento de la literatura producida en el área de estudio, la vida media podría ser en ciertos casos demasiado grande o demasiado pequeña. Por lo tanto la vida media de la literatura estaría compuesta por la tasa de obsolescencia y la tasa de crecimiento de la literatura. Sin embargo, estas afirmaciones no estaban libres de críticas, tanto así que Brookes (1970a) discute el texto de Line y afirma que "[...] un reciente análisis teórico de la relación entre la tasa de crecimiento y la tasa de obsolescencia de la literatura científica periódica realizado por Line, se basa en ciertos supuestos implícitos que es necesario hacer explícitos y después cuestionarlos. El problema teórico de medir tasas de obsolescencia cuando la literatura está creciendo es más complejo que el análisis sugerido por Line y, por lo tanto, para aclarar esta cuestión será introducido y aplicado el concepto de 'utilidad' de la literatura periódica. El problema práctico de medir la tasa de obsolescencia, que realmente depende de la muestra de una distribución geométrica también debe ser discutido, porque se puede demostrar que el tipo de técnica propuesto por Line requiere una precisión que es inalcanzable en el contexto bibliotecario".

Al año siguiente, Sandison (1971a) señaló que no había ninguna razón aparente por la cual la literatura más antigua tuviera siempre un interés decreciente. Seymour (1972a, b) realizó dos revisiones del estado del arte de las investigaciones sobre la obsolescencia de la literatura y las publicó en dos partes, una referida a las monografías y otra a las publicaciones periódicas, en las que afirmaba que los estudios de la obsolescencia eran el resultado de dos factores: la explosión de las publicaciones y la falta de espacio disponible 
en las bibliotecas. En ese mismo año Chen (1972) observó una rápida disminución de la utilización de las revistas a medida que envejecían. Sandison (1974) volvió a analizar los datos de Chen (1972) y encontró que la mayoría de las revistas mostraban un aumento en la tasa de densidad de acuerdo con la edad. Estas observaciones fueron suficientes para que Line \& Sandison (1974) definieran la obsolescencia como la disminución o caída en el tiempo de la validez o utilidad de la información. Sin embargo, los estudios y discusiones sobre este asunto continuaron con Bulick et al. (1976); Taylor (19761977); Longyear (1977); Bronmo (1978); Abramescu (1979); Gapen \& Milner (1981); Wallace (1986); McCain (1987); Heisey (1988) y otros autores.

Por esas épocas ya se habían aclarado las maneras de ejecutar los estudios sobre la obsolescencia de la literatura. Por ejemplo, Gupta (1990) observó en el campo de la física que tanto el número de citas como la tasa de densidad disminuían con la edad, mostrando una vida media de 4.9 años y ambas se ajustaban a un modelo exponencial. Aunque la obsolescencia de la literatura también puede estar influenciada por factores que no se conocen, y por eso Ravichandra Rao \& Meera (1992) investigaron la influencia de la tasa de crecimiento de la literatura en la tasa de obsolescencia y demostraron que, en el caso sincrónico, cuanto más rápido crece la literatura se vuelve obsoleta más rápidamente. También Egghe \& Ravichandra Rao (1992b) demostraron que el factor de obsolescencia definido por Brookes (1970a) como una constante independiente del tiempo realmente no es una constante, sino más bien una función estadística del tiempo, ya que en general los datos de las citas no están distribuidas exponencialmente, como lo afirmaba Brookes (1970a). En la práctica, la obsolescencia presenta un crecimiento inicial seguido de una forma de declinación exponencial, y como resultado no hay un factor de obsolescencia que sea independiente del tiempo, ya que este factor es independiente del tiempo sólo en el caso de una distribución exponencial. Egghe \& Ravichandra Rao (1992b) afirman que la distribución lognormal es el modelo que describe mejor tanto el crecimiento inicial de las citas como la declinación posterior.

Egghe (1994) estudió la combinación del crecimiento con la obsolescencia y afirmó que ambos fenómenos pueden ser estudiados con las mismas técnicas matemáticas. En el caso sincrónico, demuestra que la obsolescencia se incrementa con el crecimiento de la literatura; en el caso diacrónico, el efecto es contrario. Van Raan (2000) afirma que, sin lugar a dudas, el envejecimiento de la literatura publicada más antigua es parte de la realidad, pero la otra parte de la realidad es que en las fases iniciales de cualquier disciplina existen mucho menos documentos publicados que en los años más recientes, por lo tanto, la distribución de las referencias de acuerdo a los años siempre 
tiene una combinación de los fenómenos de envejecimiento y del crecimiento de la literatura científica. Con esta visión en mente Van Raan (2000) realizó un estudio sincrónico de las referencias de todos los artículos publicados en 1998 y cubiertos por el Science Citation Index. Los resultados revelaron que las citas iban desde 1500 hasta 1998, pero el periodo de 1500 a 1800 se caracterizó por mucho más "ruido"; es decir, citas de muy baja frecuencia a la literatura publicada, por lo cual le dio más atención a las citas de 1800 a 1998 que mostraron un crecimiento no lineal dependiente de la edad de la literatura.

Egghe \& Ravichandra Rao (2002b) analizaron la edad de las primeras referencias de los artículos publicados en la revista Journal of the American Society for Information Science (JASIS) entre 1985 y 1986. Estas primeras citas pueden ser consideradas como un análisis diacrónico de las citas, ya que son importantes en la vida de un artículo. Si en un periodo determinado el artículo es utilizado por primera vez, cambia su estatus de "no usado" por "usado", por eso se puede afirmar también que ésa es una medida de su "inmediatez" de uso. Los resultados mostraron que la distribución lognormal describe y se ajusta muy bien a la edad de las primeras referencias. Burrell (2002b) no utilizó ni empleó métodos estadísticos formales para evaluar la bondad de ajuste de las distribuciones lognormal, Weibull y log-logística, pero confió en los métodos gráficos para llevar a cabo un estudio retrospectivo (sincrónico) de las citas en un documento. Parafraseando los estudios de fiabilidad técnica en el "tiempo de vida" de los objetos mecánicos, planteó la cuestión de que las edades de las citas no son variables continuas sino variables discretas. Para ilustrar este método gráfico utilizó cinco conjuntos de datos tomados de encuestas realizadas por Egghe \& Ravichandra Rao (1992b) y Gupta (1998). El autor concluyó que el modelo de la distribución lognormal muestra más éxito describiendo el ajuste de la distribución de citas retrospectivas (sincrónico) de los documentos, confirmando estudios anteriores como los de Egghe \& Ravichandra Rao (1992b). Sobre este tema volvió a hacer hincapié en artículos posteriores (Burrell, 2002b, 2003a, b) afirmando que parecía razonable pensar que cuando se citan artículos, no siempre es posible citar los artículos más nuevos, porque todavía no se sabe mucho sobre ellos o porque aún no fueron incorporados en el conocimiento de los investigadores, entonces la mayor parte de las citas serían a artículos de edad mediana, que constituirían la mayor parte de las referencias y las que tienen más probabilidades de ser citadas; luego, los artículos más antiguos serían citados selectivamente para proporcionar una perspectiva histórica en las investigaciones realizadas.

Ahmed, Johnson, Oppenheim \& Peck (2004) llevaron a cabo un estudio diacrónico del artículo sobre la estructura de la doble hélice del ADN, publicado en 1953 por Watson y Crick (1953). Utilizaron los volúmenes impresos 
del Science Citation Index de 1961 a 1980, y el Web of Science del ISI, para el periodo de 1981 a 2002. Observaron 2061 citas, con una media de 49 citas por año. Los autores concluyeron que este artículo sigue siendo continuamente citado 50 años después de su publicación y no encontraron ninguna explicación sobre las razones por las cuales sigue siendo citado. Glänzel (2004) ofreció una visión panorámica del envejecimiento desde la perspectiva de la teoría de la fiabilidad, representando los diferentes aspectos que pueden ser analizados por ambos métodos, sincrónico y diacrónico. Si las citas de un documento son consideradas como una forma del uso de la información en el proceso de la comunicación científica, la "fiabilidad técnica" de un artículo científico expresa la realización de su función intencionada; es decir, que sea leído, que tenga algún impacto en la investigación científica y que su fiabilidad pueda ser medida por las citas. Como cualquier tecnología que no funciona correctamente, puede considerarse que un artículo nunca citado solamente desempeñó satisfactoriamente su función pretendida en el momento de su publicación. Por lo tanto, el concepto de "fiabilidad técnica" implica una perspectiva diacrónica (prospectiva).

Más recientemente Zafrunnisha \& Reddy (2010) estudiaron la obsolescencia de las tesis de doctorado en psicología de 1963 a 2005 en cuatro universidades de la India. Encontraron que la vida-media para esta literatura es de 14 años para los artículos y 19 años para los libros.

\section{MODELO TEÓRICO}

La obsolescencia de la literatura es medida a través de la técnica del análisis de citas. Esta técnica supone que existe una relación de asociación entre el documento citado y el documento citante. Persistente y constantemente los autores citan los documentos más actuales en desmedro de los documentos más antiguos que son relegados en su frecuencia de uso (citas), y son condenados a la obsolescencia, porque pocos o nadie los usan (citan). Esa idea de relación entre los documentos citantes y los documentos citados conforman el modelo teórico de esta investigación y se plasma en la Figura 1. En este artículo se usará como base la literatura publicada sobre "Ley de Lotka" desde 1926 hasta 2010. Hasta 2010 se publicaron 663 documentos en diferentes idiomas, de los cuales se tomaron como base sólo las citas de 86 documentos publicados entre 2007 y 2010.

La lógica de elaboración de nuevos documentos de investigación sugiere que cualquier autor que en 2008 haya escrito un artículo sobre la Ley de Lotka tuvo a su disposición 605 documentos producidos hasta el año anterior 


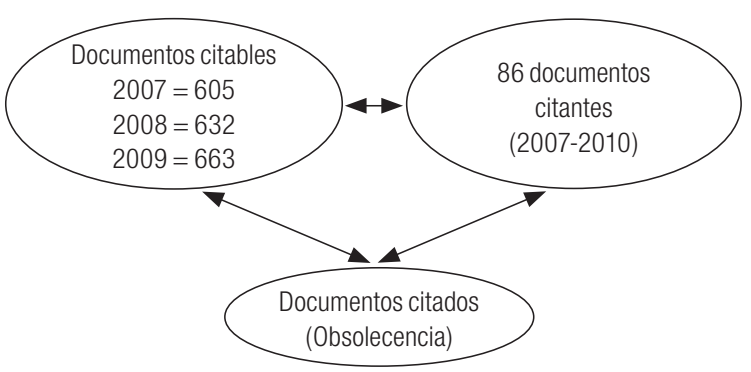

Figura 1. Modelo teórico de la investigación.

(2007) para ser recuperados, revisados y citados. Igualmente cualquier autor que en 2009 haya escrito un artículo sobre la Ley de Lotka tuvo a su disposición los 605 documentos producidos hasta el 2007 más los 27 documentos publicados en 2008; es decir, un total de 632 publicaciones para ser recuperados, revisados y citados. Similarmente, un autor que en 2010 haya escrito un artículo sobre la Ley de Lotka tuvo a su disposición los documentos producidos hasta el 2007 (605 documentos), más los producidos en el 2008 (27 documentos), más los producidos en el 2009 (28 documentos) haciendo un total de 663 documentos para ser recuperados, revisados y citados. Es en esta literatura potencialmente citable que se pretende medir la obsolescencia de la literatura. Se espera que las citas a los documentos anteriores, si existe obsolescencia de la información, sean de forma exponencial como han sido observadas en otras investigaciones y listadas en la revisión de la literatura. La ausencia de esta forma de dispersión de la "nube de puntos" en los datos observados indicaría que no existe obsolescencia y la causa de esta forma de dispersión de la literatura citada tendría otras variables causales desconocidas y no estudiadas en este trabajo.

\section{Metodología}

Para identificar los documentos publicados se hizo una búsqueda en todas las bases de datos de DIALOG, con los términos Lotka?(5n)Law?. Esta estrategia de búsqueda produjo un total de 515 registros, que después de la depuración de los duplicados y falsas recuperaciones fueron acumulados en un total de 457 referencias bibliográficas. Esas 457 referencias fueron trasladadas a Pro-Cite 5.0 para la elaboración de una base de datos específica sobre este asunto. También fueron realizadas búsquedas en el Information Science Abstract (ISA), Library Literature (LL) y Library and Information Science Abstract (LISA), Web of Knowledge, Scopus y bases de datos Latinoamericanas como Infobila de México y LICI del Instituto Brasileiro de Informação em Ciência 
e Tecnologia (IBICT). Igualmente, se consultaron bases de datos chinas con China Academic Journals via EastView Online Services y Japonesas via CiNii: Citation Information by National Institute of Informatics, Japanese Scholarly \& Academic Information. De esa manera se reunió una bibliografía analítica sobre la Ley de Lotka que lista un total de 691 referencias bibliográficas e incluye artículos de revistas, capítulos de libros, comunicaciones presentadas en congresos, folletos y cartas dirigidas a los editores de revistas especializadas en bibliotecología y ciencia de la información producidas y publicadas desde 1926 hasta diciembre de 2011. ${ }^{1}$ De estos 691 documentos contenidos en la base de datos en Pro-Cite 5.0, se tomaron como documentos-base solamente los publicados en el 2008 (27 documentos), 2009 ( 31 documentos) y 2010 (28 documentos), haciendo un total de 86 documentos, de los cuales se aislaron sólo las citas referidas a los 663 documentos publicados entre 1926 y 2007.

Para medir los datos de las citas recogidas se consideró el modelo exponencial que en la literatura publicada es sugerido como el que describe mejor la distribución observada de las citas. El decrecimiento exponencial representa una reducción de la población en una proporción fija en cada unidad de tiempo. También se asume una tasa constante de descenso con un límite de decrecimiento no definido. El modelo no sólo ofrece una tasa media de decrecimiento, sino que también proporciona una tasa de duplicación de ese decrecimiento; es decir, una tasa en la cual el tamaño de la población se reduce a la mitad. Según Egghe \& Ravichandra Rao (1992b: 201), sólo "el factor de obsolescencia $a$ debe ser determinado, ya que tanto la vida media como el factor de utilidad, son simples funciones de $a$ ". Matemáticamente esta función se representa como:

$$
c(t)=\theta e^{-\theta t}
$$

para $t \geq 0$ y donde el parámetro $\theta>0$.

Con base en esta propuesta, el factor de obsolescencia $a$ se define como

$$
a(t)=a=\frac{c(t+1)}{c(t)}
$$

En el caso de la ecuación (2), a ( $t$ ) es independiente de $t$, de modo que

$$
a(t)=\frac{\theta e^{-\theta(t+1)}}{\theta e^{-\theta t}}=e^{-\theta}
$$

1 El autor mantiene una Base de Datos Bibliográfica en Pro-Cite 5.0 sobre este asunto que acpermanentemente. 
que Egghe \& Ravichandra Rao (1992a) definen como el factor de envejecimiento

$$
a=e^{-\theta}
$$

Sin embargo, en la práctica ocurren muchas fluctuaciones en los valores de las citas con cero años de obsolescencia o con los primeros cinco años de obsolescencia. La mejor manera de superar este problema fue propuesta por Brookes (1973). Entonces, asumiendo como verdaderas las ecuaciones (1) y (2), se puede escribir que

$$
c(t)=\theta a^{t}
$$

Dejando que $m$ indique el número total de citas a las publicaciones que tienen $t$ años de edad, se tiene que

$$
\begin{aligned}
& m=\theta a^{t}+\theta a^{t+1}+\ldots \\
& m=a^{t}\left(\theta+\theta a+\theta a^{2}\right) \\
& m=a^{t} T
\end{aligned}
$$

donde $T$ denota el número total de citas. Por lo tanto,

$$
a=\left(\frac{m}{T}\right)^{1 / t}
$$

Sin embargo, en general los datos de las citas no se ajustan a la ecuación (1) y muestran un trazado diferente. Los trazados de los datos encontrados con mayor frecuencia en las investigaciones realizadas muestran un crecimiento inicial de las citas seguido de una forma de decrecimiento exponencial. Como resultado de ello, "no hay una manera de encontrar un factor de envejecimiento $a$ independiente de $t: a$ solamente es independiente de $t$ en el caso de la distribución exponencial" (Egghe \& Ravichandra Rao, 1992b: 203). Por esta razón, estos autores recomiendan el uso de la vida-media y también señalan que el factor de utilidad total $U$ está directamente relacionada con el factor de envejecimiento mediante la fórmula:

$$
U=\frac{1}{1-a}
$$

para $t \geq 0$. 
Ciertamente la frecuencia de citas de la literatura publicada en cualquier área del conocimiento disminuye a medida que pasa el tiempo, de modo que cuanto más antiguo es el documento, sus posibilidades de ser citado serán menores, pero no sabemos la forma de esta disminución. Por lo tanto, al estudiar la obsolescencia de la literatura se postula una relación entre la edad de la literatura (variable independiente), medida en años, y el número de citas (variable dependiente), medido en volumen de citas producidas. Se supone que esta relación puede ser estadísticamente modelable, de modo que para evaluar el ajuste del modelo se construye un gráfico de la dispersión de la "nube de puntos" sobre la base de los datos observados. Esto nos permite evaluar si existe cierta regularidad en la distribución de frecuencias observadas, y cuando esa regularidad se asemeja a la curva que se muestra en el gráfico elaborado de la "nube de puntos", se intenta ajustar esa curva a través de una regresión no lineal. En la distribución exponencial, lo que se pondrá a prueba es si la frecuencia de las citas de los documentos según los años $(t)$ proviene de una distribución no lineal. Es decir, la probabilidad de que la frecuencia de citas en la muestra sea igualmente probable para todas las citas en la misma situación. Como se espera una alta correlación entre las variables dependientes e independientes, esa correlación será explorada mediante el coeficiente de correlación de Pearson. El análisis de la varianza y el cálculo de la pendiente y la interceptación de la distribución exponencial se realizaron por el método de estimación de la curva de regresión no lineal, utilizando el software estadístico SPSS 17.0 y Mathematica 5.0 para Windows.

\section{Resultados}

A pesar de los que afirman que las investigaciones en esta área "han sido ampliamente analizadas y demostradas desde el año 1926, por lo que actualmente se puede considerar que forman parte del conocimiento en bibliometría, así que poco se puede aportar de nuevo sobre él [...] y que ya no son de beneficio ni se publican más trabajos sobre este asunto" (Referí de la Revista Española de Documentación Científica, 2011), el interés por investigar en este campo sigue en crecimiento permanente, tanto que en estos tres años (20082010) se han incorporado a este asunto el $15 \%$ del total de documentos existentes hasta el 2007. No en vano Urbizagástegui (2009: 120) afirma que esta área crece a una tasa anual del 7 \% y duplica su volumen cada 10.2 años. Sin embargo, no se ha estudiado la obsolescencia de esta literatura publicada, ni la de los idiomas en que se escriben, ni tampoco la de las citas efectuadas a estos documentos. Naturalmente el idioma del documento publicado y el 
idioma de las citas referenciadas en las publicaciones tienen obvias implicaciones en la obsolescencia de la literatura. La Tabla 1 muestra los idiomas de los 86 documentos en estudio según los años de publicación de los mismos.

Tabla 1. Idiomas de los documentos publicados según los años.

\begin{tabular}{|c|c|c|c|c|c|c|c|}
\hline Años & Inglés & Portugués & Chino & Español & Turco & Alemán & Total \\
\hline 2008 & 16 & 5 & 3 & 2 & 1 & - & 27 \\
\hline & $(59.3)$ & $(18.2)$ & $(11.1)$ & $(7.4)$ & $(3.7)$ & - & $(100.0)$ \\
\hline 2009 & 21 & 4 & 3 & 2 & - & 1 & 31 \\
\hline & $(67.7)$ & $(12.9)$ & $(9.7)$ & $(6.5)$ & - & $(3.2)$ & $(100.0)$ \\
\hline 2010 & 26 & 1 & - & 1 & - & - & 28 \\
\hline & $(92.9)$ & $(3.4)$ & - & $(3.4)$ & - & - & $(100.0)$ \\
\hline Total & 63 & 10 & 6 & 5 & 1 & 1 & 86 \\
\hline & $(73.3)$ & $(11.6)$ & $(7.0)$ & $(5.8)$ & $(1.2)$ & $(1.2)$ & $(100.0)$ \\
\hline
\end{tabular}

* Las cifras entre paréntesis indican porcentajes.

El idioma inglés $(73 \%)$ domina la producción de documentos, seguido de las publicaciones en portugués (12\%), chino (7\%) y español (6 \%). Las publicaciones en turco y alemán son poco significativas y representan apenas el $1 \%$ del total de documentos publicados en el periodo de investigación. Consistentemente, a través de los años se publican tres veces más documentos en inglés que en el resto de los idiomas sobre la Ley de Lotka.

La Tabla 2 muestra los idiomas citados por los 63 documentos publicados en inglés en el periodo de estudio (2008-2010). Consistentemente, a través de los años quienes publican en inglés sólo citan documentos en inglés. Por ejemplo, en el año 2008 se publicaron 16 documentos en inglés y estos documentos citaron 358 veces otros documentos también publicados en inglés que representaron un $98 \%$ de todo lo citado ese año. Igualmente, en el año 2009 se citaron 662 veces documentos escritos y publicados en inglés que representaron el $94.4 \%$ de todo lo citado ese año. De los 26 documentos publicados en inglés en el año 2010, todos ellos (100 \%) sólo citaron otros documentos publicados también en inglés. En general, en los tres años analizados, $97 \%$ de las citas son de documentos publicados en inglés.

Tabla 2. Citas de los documentos publicados en inglés.

\begin{tabular}{|c|c|c|c|c|c|c|c|c|c|}
\hline Años & $\begin{array}{c}\text { Núm. } \\
\text { docs. }\end{array}$ & $\begin{array}{c}\text { Citas en } \\
\text { inglés }\end{array}$ & $\begin{array}{c}\text { Citas en } \\
\text { francés }\end{array}$ & $\begin{array}{c}\text { Citas en } \\
\text { alemán }\end{array}$ & $\begin{array}{c}\text { Citas en } \\
\text { holandés }\end{array}$ & $\begin{array}{c}\text { Citas en } \\
\text { español }\end{array}$ & $\begin{array}{c}\text { Citas en } \\
\text { portugués }\end{array}$ & $\begin{array}{c}\text { Citas en } \\
\text { catalán }\end{array}$ & $\begin{array}{c}\text { Total de } \\
\text { citas }\end{array}$ \\
\hline 2008 & 16 & 358 & 3 & 1 & 1 & 1 & 1 & - & 365 \\
\hline & & $(98.0)$ & $(0.82)$ & $(0.3)$ & $(0.3)$ & $(0.3)$ & $(0.3)$ & - & $(100.0)$ \\
\hline 2009 & 21 & 625 & 1 & - & - & 30 & 3 & 3 & 662 \\
\hline
\end{tabular}




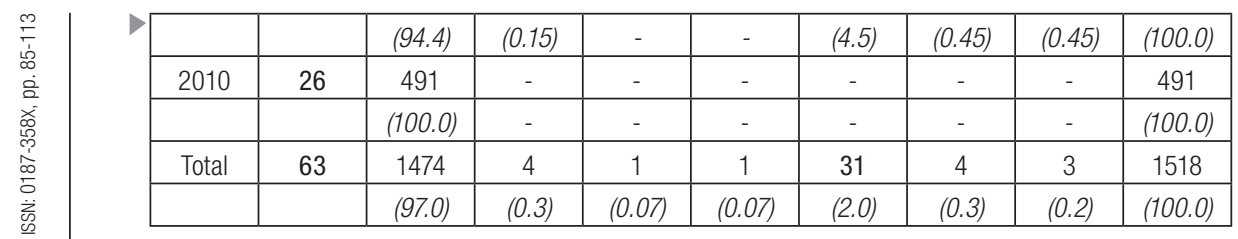

* Las cifras entre paréntesis indican porcentajes.

En esta Tabla 2 se observa también un comportamiento anómalo en el año 2009. En ese año se produjeron 30 citas en español, pero estas citas proceden de un único documento publicado en inglés cuyo autor reside y trabaja en una región donde se habla español, pero se ve forzado a publicar en inglés porque cree que de esa manera su documento será más visible y ganará una amplia difusión internacional; en otras palabras, está buscando los caminos "para alcanzar una visibilidad más justa de nuestra producción científica en el área de ciencia de la información” (Miranda, 1981; 1982).

Tres variables pueden estar en juego aquí: una puede ser la endogamia de quienes publican en inglés estando seguros de que "el lenguaje de la ciencia es el inglés" (Price, 1971; Baldauf, 1986) y no se preocupan por consultar documentos en lenguas diferentes al inglés porque menosprecian lo publicado en otros idiomas. Otra variable puede ser la carencia de familiaridad o el desconocimiento de idiomas extranjeros, parecería que quienes tienen como lengua nativa el inglés no conocen otra lengua diferente al inglés y esto refuerza el comportamiento endogámico. La última variable puede ser la accesibilidad a los documentos producidos en otros idiomas diferentes al inglés, pero dado que este asunto es mayoritariamente publicado por especialistas del campo de la bibliotecología y la ciencia de la información que conocen todos los mecanismos para recuperar la información deseada, no hay razón aparente que explique ese mecanismo de acceso al documento. Es difícil creer que sea la accesibilidad uno de los factores que impulsa la poca preocupación por citar otros documentos publicados en idiomas diferentes al inglés. Tal vez se pudiera aducir que lo implicado fuera el costo pero tampoco esa variable parece factible. Por lo tanto, se refuerza la tesis de la endogamia.

Esto no parece ocurrir con aquellos que publican documentos en otros idiomas. La Tabla 3 muestra los documentos publicados en portugués. Los 10 documentos publicados en el periodo de la investigación realizaron un total de 353 citas a otros documentos publicados de 1926 a 2007. De estas 353 citas, $44 \%$ fueron a documentos publicados en portugués y $52 \%$ a documentos publicados en inglés. Solamente en el año 2010 hubo una alta concentración de las citas a documentos en el mismo idioma portugués (86 $\%)$. El acompañamiento a las publicaciones en inglés parece ser permanente, 
consistente y a la par del portugués. El acompañamiento a publicaciones en otros idiomas es poco significativo, es decir, el seguimiento de las publicaciones en español, francés y alemán es casi nulo. Claramente, quienes publican en portugués, además de lo que se publica en el contexto cultural nacional, acompañan la producción de quienes lo hacen en inglés.

Tabla 3. Citas de los documentos publicados en portugués.

\begin{tabular}{|c|c|c|c|c|c|c|c|}
\hline Años & $\begin{array}{c}\text { Núm. } \\
\text { docs. }\end{array}$ & $\begin{array}{c}\text { Citas en } \\
\text { portugués }\end{array}$ & $\begin{array}{c}\text { Citas en } \\
\text { inglés }\end{array}$ & $\begin{array}{c}\text { Citas en } \\
\text { español }\end{array}$ & $\begin{array}{c}\text { Citas en } \\
\text { francés }\end{array}$ & $\begin{array}{c}\text { Citas en } \\
\text { alemán }\end{array}$ & $\begin{array}{c}\text { Total de } \\
\text { citas }\end{array}$ \\
\hline 2008 & 5 & 85 & 62 & 4 & - & - & 151 \\
\hline & & $(56.3)$ & $(41.0)$ & $(2.6)$ & - & - & $(100.0)$ \\
\hline 2009 & 4 & 39 & 118 & 7 & 2 & - & 166 \\
\hline & & $(23.5)$ & $(71.0)$ & $(4.2)$ & $(1.2)$ & - & $(100.0)$ \\
\hline 2010 & 1 & 31 & 3 & - & - & 2 & 36 \\
\hline & & $(86.1)$ & $(8.3)$ & - & - & $(5.5)$ & $(100.0)$ \\
\hline Total & 10 & 155 & 183 & 11 & 2 & 2 & 353 \\
\hline & & $(44.0)$ & $(51.9)$ & $(3.1)$ & $(0.56)$ & $(0.56)$ & $(100.0)$ \\
\hline
\end{tabular}

* Las cifras entre paréntesis indican porcentajes.

Este comportamiento es similar entre aquellos que publican en español. De los 5 documentos publicados en este idioma en el periodo de investigación (ver la Tabla 4), 37 \% son citas a documentos del propio idioma; $58 \%$ a documentos publicados en inglés; $3 \%$ a documentos en francés y $2 \%$ a documentos en portugués. En este caso tampoco el comportamiento endogámico parece jugar un papel preponderante. Con excepción del 2009, una quinta parte de lo que se cita está dirigido a documentos publicados en inglés. Por lo tanto, quienes publican en español también están atentos a lo que se publica en su propio idioma y a los documentos publicados en inglés.

Tabla 4. Citas de los documentos publicados en español.

\begin{tabular}{|c|c|c|c|c|c|c|}
\hline Años. & $\begin{array}{c}\text { Núm. } \\
\text { docs. }\end{array}$ & $\begin{array}{c}\text { Citas en } \\
\text { español }\end{array}$ & $\begin{array}{c}\text { Citas en } \\
\text { inglés }\end{array}$ & $\begin{array}{c}\text { Citas en } \\
\text { francés }\end{array}$ & $\begin{array}{c}\text { Citas en } \\
\text { portugués }\end{array}$ & $\begin{array}{c}\text { Total de } \\
\text { citas }\end{array}$ \\
\hline 2008 & 2 & 18 & 6 & 4 & - & 28 \\
\hline & & $(64.3)$ & $(21.4)$ & $(14.3)$ & - & $(100.0)$ \\
\hline 2009 & 2 & 9 & 59 & - & 2 & 70 \\
\hline 2010 & 1 & $(13.0)$ & $(84.0)$ & - & $(3.0)$ & $(100.0)$ \\
\hline & & $(78.3)$ & $(21.7)$ & - & - & 23 \\
\hline
\end{tabular}




\begin{tabular}{|c|c|c|c|c|c|c|}
\hline Total & 5 & 45 & 70 & 4 & 2 & 121 \\
\hline & & $(37.2)$ & $(57.9)$ & $(3.3)$ & $(1.7)$ & $(100.0)$ \\
\hline
\end{tabular}

* Las cifras entre paréntesis indican porcentajes.

Esta comprobación trae consecuencias desastrosas para aquellos autores que publican en idiomas diferentes al inglés. Como la obsolescencia de la literatura es medida a través de las citas que se hacen de los documentos, y como los que publican en inglés sólo citan documentos publicados en inglés y también los documentos publicados en este idioma son los más numerosos (73\%), esto significa que los documentos publicados en otros idiomas nacen sin posibilidades de ser leídos por esa "comunidad internacional" a la que se le exige una "visibilidad más justa" (Miranda, 1981; 1982). Es decir, quienes no publican en inglés escriben para un público que no va a leer ni va a citar esos documentos porque no citan documentos en otros idiomas diferentes al inglés, ya sea porque no tienen familiaridad con idiomas diferentes al inglés, ya sea por la endogamia que los caracteriza. Entonces la obsolescencia, si existe, sólo va a existir entre los documentos publicados en inglés porque los documentos publicados en otros idiomas nacen obsoletos incluso antes de ser escritos. Por lo tanto, el consumo de la información que se produce en idiomas diferentes del inglés estará restringido a las regiones donde aquélla se produce; es decir, a un consumo local entre aquellos socializados en el idioma del autor.

Los 86 documentos de la muestra fueron producidos por 95 autores que en el periodo de la investigación recibieron un total de 416 citas. Para identificar a los autores más citados se usó la Ley de Bradford que produjo tres zonas consistentes. ${ }^{2}$ La división zonal puede ser observada en la Tabla 5. El

2 Uno de los evaluadores de este artículo afirma que "Hay serias dudas sobre el uso de la ley de Bradford para calificar la distribución de las citas de los autores del área: no se demuestra en el texto ni se apoya en literatura contrastada, que las citas sigan un modelo similar al de la distribución de la literatura en las fuentes en que se publican. Que la ley se pueda aplicar no implica que tenga sentido aplicarla. Hay métodos alternativos para describir tales distribuciones, como el uso de cuartiles, deciles o representaciones gráficas como diagramas del tipo Box-plot" (Dictaminador núm. 1). Sin entrar en polémicas innecesarias, es de sentido común no usar "cuartiles, deciles o representaciones gráficas como diagramas del tipo Box-plot" para identicar a los autores más citados. Y ya que este evaluador demanda "literatura contratada" menciono solamente a Hubert (1978) y Yablonsky (1980), quienes discutieron la equivalencia de la Ley de Bradford, la Ley de Lotka y la Ley de Zipf, pero quienes derivaron una relación funcional común entre estas tres leyes bibliométricas fueron Chen \& Leimhkuler (1986). Por lo tanto es lícito aplicar una u otra ley a las citas, sólo se necesita creatividad. Este mismo evaluador demanda la inclusión de los datos de las citas, pero como éstas van de 1926 a 2010, ocupan tres páginas y harían este artículo más extenso de lo permitido por la revista que ya está solicitando recorte de la extensión del mismo. Así, los interesados en los datos los pueden solicitar directamente al autor y con mucho gusto éstos le serán proporcionados. 
núcleo central está formado por 6 autores citados en media 24 veces; la zona de frontera consta de 16 autores, citados en media 8.5 veces, y hay una zona de dispersión conformada por 73 autores citados en media 2 veces en los tres años estudiados. Hasta el año 2010 un total de 728 autores habían participado en la publicación de por lo menos un artículo, pero solamente $13 \%$ de los autores activos en este campo fueron citados. En otras palabras, $87 \%$ de los autores productores de literatura sobre este asunto no son leídos ni son citados. Esto significa que apenas $0.8 \%$ de los autores activos en este campo han sido citados 19 o más veces en el periodo investigado y la mayoría de estos autores escriben y publican en inglés.

Tabla 5. Autores según la División zonal de Bradford.

\begin{tabular}{|c|c|c|c|}
\hline Zonas & Núm. de autores & Núm. de citas & Media de citación \\
\hline 1 & 6 & 144 & 24.0 \\
\hline & $(6.3)$ & $(34.6)$ & \\
\hline 2 & 16 & 136 & 8.5 \\
\hline & $(16.8)$ & $(32.6)$ & \\
\hline 3 & 73 & 136 & 1.9 \\
\hline & $(76.8)$ & $(32.6)$ & \\
\hline Total & 95 & 416 & 4.4 \\
\hline
\end{tabular}

* Las cifras entre paréntesis indican porcentajes.

Si se considera a los autores que han sido citados una o más veces, se observa que apenas $6 \%$ de ellos son citados continuamente por un tercio de los documentos, pero dos tercios de los documentos citan sólo a $23 \%$ de los autores observados. Es decir que, en este campo, apenas $23 \%$ de los autores observados tienen posibilidades de continuar sobreviviendo, escapar a la obsolescencia y ser visibles por una comunidad llamada "internacional". De los 728 autores activos en este asunto sólo 95 de ellos fueron citados una o más veces entre 2007 y 2010. También sólo 22 de ellos fueron citados más de 4 veces. Estos 22 autores son los que tienen mayores posibilidades de afianzarse en el campo, de continuar sobreviviendo y de escapar a la obsolescencia. La Figura 2 muestra el trazado en escala semi-logarítmica de los datos observados. El trazado muestra claramente un comportamiento bradfordiano con una porción cóncava inicial para después convertirse en una línea recta indicando la dispersión de los autores. El núcleo está conformado por los 6 primeros autores listados en la Tabla 6. 


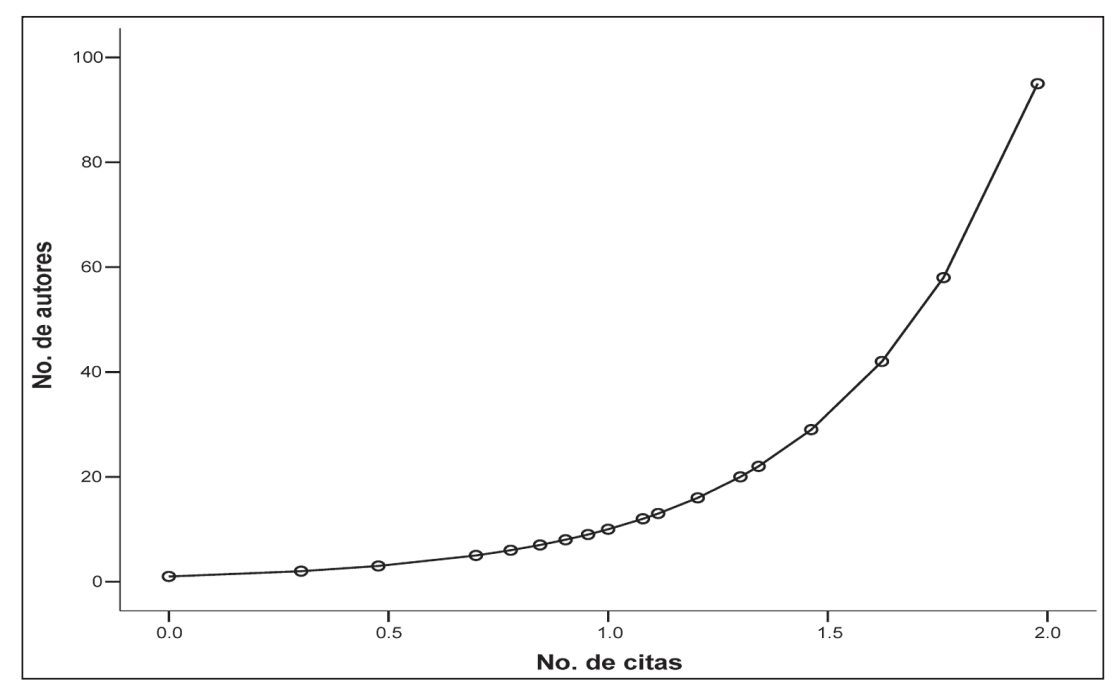

Figura 2. Trazado de los autores según las citas.

A pesar de que ya fue probado que el modelo del cuadrado inverso propuesto por Lotka (1926) no soporta una prueba estadística y de que se hayan propuesto otros modelos alternativos que predicen mejor la productividad de los autores, como el modelo del poder inverso generalizado, el modelo Gauss Poison inverso generalizado, Poisson lognormal, etc., este autor aún sigue siendo fuertemente citado. El mecanismo de incorporación por obliteración parece no operar en el caso de Alfred J. Lotka. Lo mismo puede decirse del texto de Price, pues el capítulo "Galton revisited" de su libro Little Science, Big Science (Price, 1963) es el tercero más citado (17 veces) seguido del artículo de Price (1976), que es citado 5 veces.

Tabla 6. Autores más citados.

\begin{tabular}{|c|c|}
\hline Autores del Núcleo & Núm. de citas \\
\hline Lotka, Alfred J & 39 \\
\hline Pao, Miranda Lee & 24 \\
\hline Price, John Derek de Solla & 22 \\
\hline Rousseau, Ronald & 20 \\
\hline Nicholls, Paul Travis & 20 \\
\hline Egghe, Leo & 19 \\
\hline Autores en la zona de frontera & Núm. de citas \\
\hline Potter, William Gray & 16 \\
\hline Vlachý, Jan & 14 \\
\hline Urbizagástegui Alvarado, Rubén & 12 \\
\hline
\end{tabular}




\begin{tabular}{|c|c|}
\hline Chung, Kee H. & 11 \\
\hline Patra, Swapan Kumar & 10 \\
\hline Kretschmer, Hildrum & 10 \\
\hline Bookstein, Abraham & 8 \\
\hline Newby, Gregory B. & 7 \\
\hline Rowlands, lan & 6 \\
\hline Gupta, B. M. & 6 \\
\hline Schorr, Alan Edward & 5 \\
\hline Nath, Ravinder & 5 \\
\hline Sen, B. K., Che & 4 \\
\hline Radhakrishnan, T. & 4 \\
\hline Kuperman, Victor & 4 \\
\hline Huber, John C. & 4 \\
\hline
\end{tabular}

La segunda más citada es Miranda Lee Pao, pero lo que se cita de esta autora son 4 documentos diferentes. El documento más citado es Pao (1985) que es citado 14 veces y luego Pao (1986) que es citado 8 veces y otros dos documentos con una cita cada uno. De Ronald Rousseau se citan 5 documentos diferentes siendo el documento de Rousseau \& Rousseau (2000) el más citado (13 veces), Rousseau (1992) 2 veces, Rousseau (1993) 2 veces y otros dos documentos con una cita cada uno. De Paul Travis Nicholls se citan 4 artículos diferentes; Nicholls (1989) 9 veces, Nicholls (1986) 9 veces y Nicholls (1987, 1988) 2 veces cada uno. De Leo Egghe se citan 14 documentos diferentes, uno 4 veces (Egghe, 2005a), otro 2 veces (Egghe, 2005b) y el resto una vez cada uno. En general, los autores del núcleo tienen diferentes cantidades de publicaciones con diferentes frecuencias de citación. Este mismo patrón de comportamiento se repite con los autores de la zona 2 ubicados en la zona de frontera. En otras palabras, a más documentos publicados, mayores posibilidades de citas.

La Figura 3 muestra la distribución de las citas ordenadas de las más actuales a las más antiguas. A pesar de observarse un crecimiento de las citas en los primeros 5 años no se observa un patrón de obsolescencia definido, pues es notoria la oscilación de las citas representadas en el gráfico de barras. Por ejemplo, las barras más elevadas representan, respectivamente, las publicaciones más citadas de autores como Alfred Lotka, Miranda Lee Pao, John Derek de Solla Price, Ronald Rousseau, Paul Travis Nicholls y Leo Egghe. Si no fuera por estos autores las citas se agruparían en cuatro sectores bien diferenciados; tres de estos grupos bastante cohesionados y el último muy disperso. 


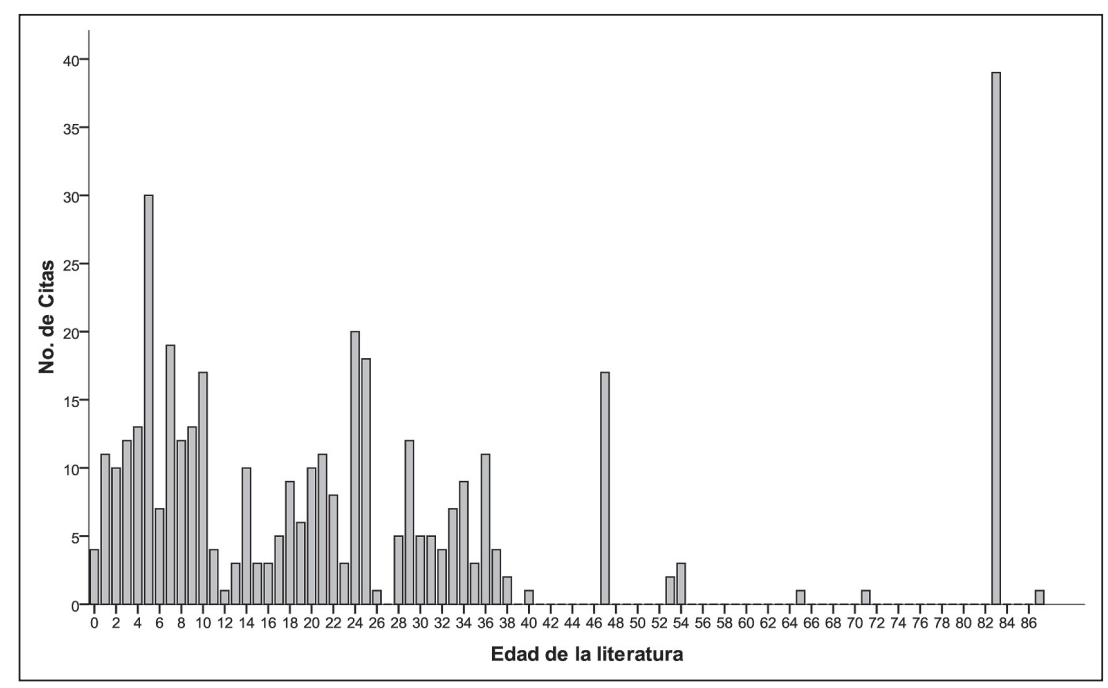

Figura 3. Distribución de las citas según la edad de la literatura.

No existe un patrón de obsolescencia definido. Un patrón de obsolescencia exponencial mostraría una caída desde el punto inicial descendiendo constantemente hasta el final de la distribución. Esa caída no se muestra claramente en el gráfico de barras mostrada en la Figura 3. Esa caída debería ser muy parecida al trazado de la Figura 4.

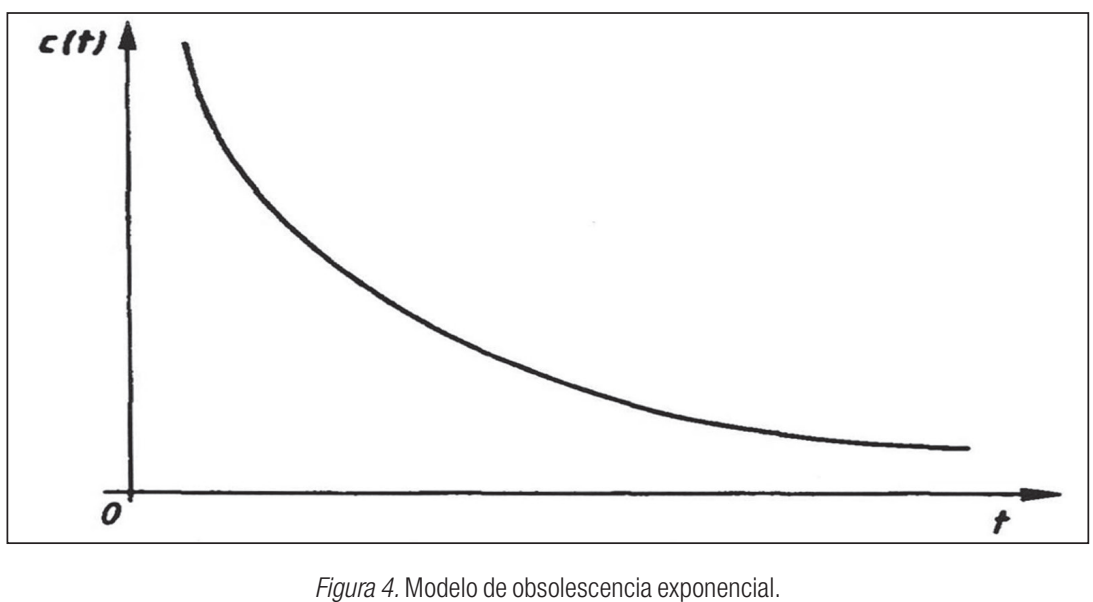

Un patrón de obsolescencia lognormal mostraría una subida inicial muy lenta, para luego llegar a un tope máximo en los primeros cuatro o cinco años. A partir de ese punto máximo de inflexión comenzaría a descender 
hasta el final de la distribución. Ese crecimiento inicial, a pesar que parece mostrarse en los primeros cinco años, es muy irregular y no conforma un proceso de obsolescencia lognormal real y consistente en la Figura 3. La Figura 5 ejemplifica lo que debería ser un modelo de decrecimiento lognormal.

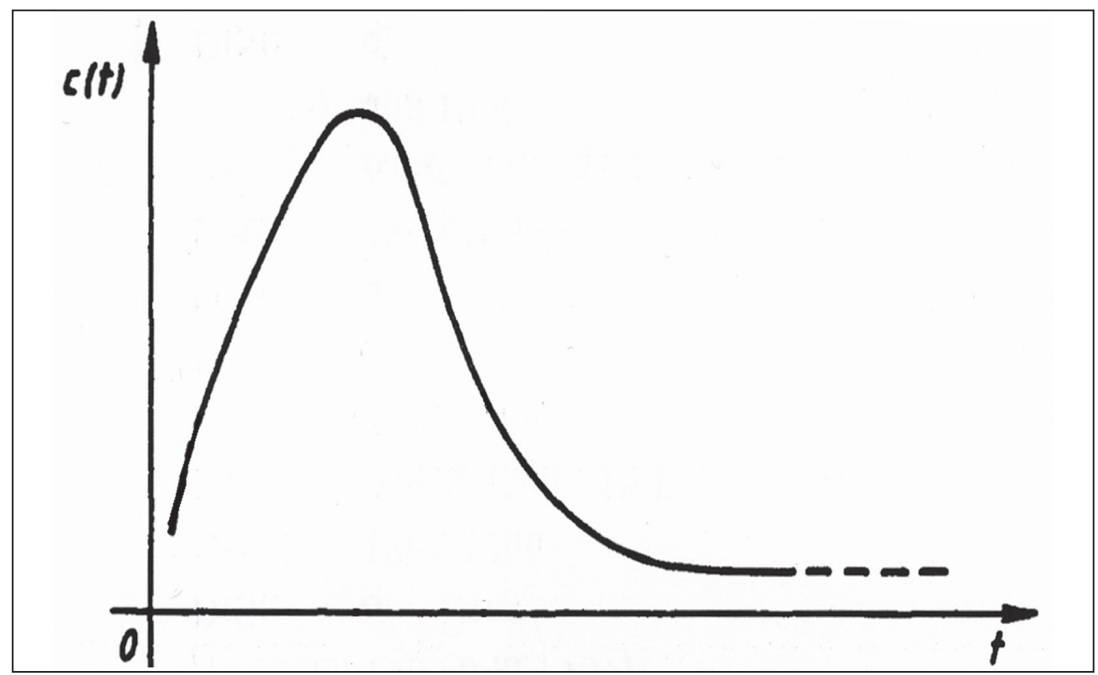

Figura 5. Modelo de obsolescencia lognormal.

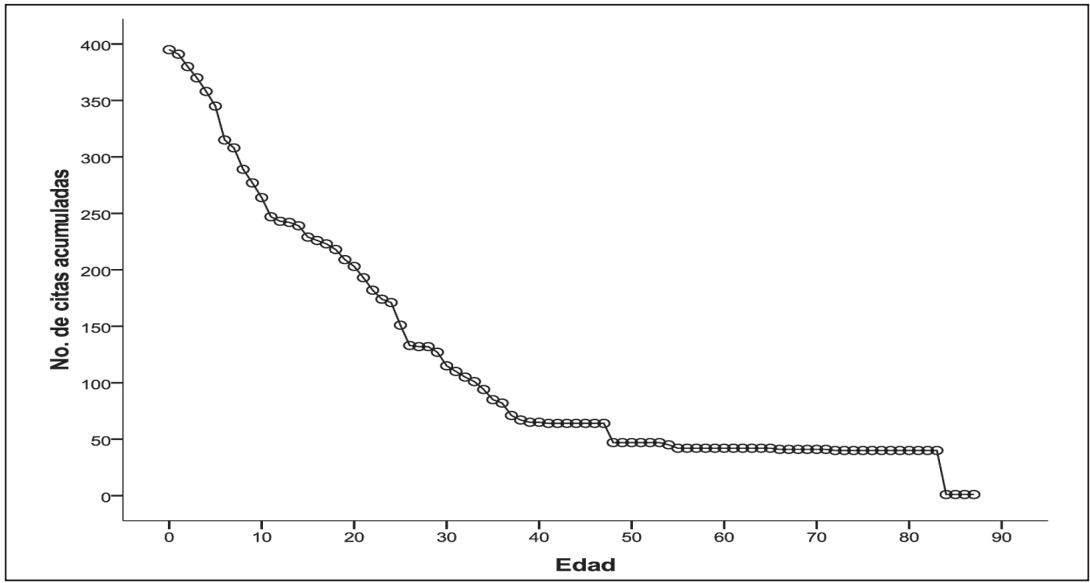

Figura 6. Distribución de la citas acumuladas observadas según la edad.

La distribución de las citas acumuladas por edades parece producir una obsolescencia exponencial (Figura 6). Puede observarse que existe una caída de forma exponencial conforme la literatura citada envejece y es evidente 
una tendencia en la que los altos valores de las frecuencias de las citas están asociados con la edad de la literatura más reciente. Los valores más bajos de las frecuencias de las referencias están asociados con las edades más antiguas de la literatura citada.

Usando el software estadístico SPSS 17.0 para Windows se estimó la función exponencial no-lineal propuesta por Egghe \& Ravichandra Rao (1992b). Se estimó una regresión no-linear que produjo un $r^{2}$ ajustado estimado de 0.985 indicando que más o menos $98 \%$ de las variaciones en la frecuencia de citas son debidas o dependen de las variaciones ocurridas en la edad de la literatura. El valor estimado de $C$ fue igual a 407.069 y el de $g$ fue igual a -0.961. Esos valores produjeron un error estándar asintótico de 5.098 para $g$ y de 0.001 para $C$. La ecuación de obsolescencia estimada por el método de máxima probabilidad no-linear con 86 grados de libertad es entonces

$$
C(t)=407.069(-0.961)^{\mathrm{t}}
$$

indicando que la literatura decrece a una tasa de $3.9 \%$ al año y alcanza una tasa de envejecimiento medio a la edad de 17.4 años.

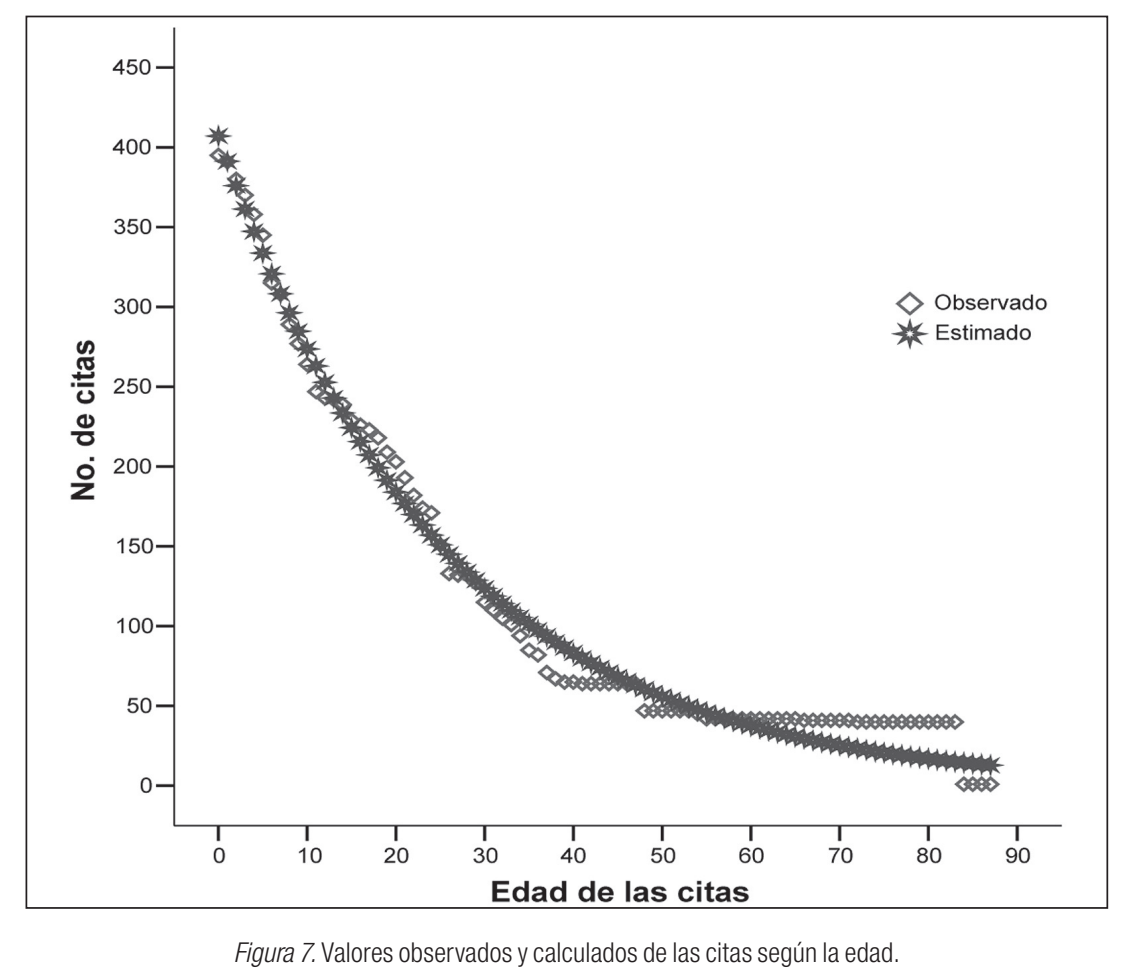


La Figura 7 muestra los valores observados y estimados de la frecuencia acumulada versus la edad de las citas. Esta figura proporciona una indicación visual de la forma como la frecuencia de citas y la edad de la literatura co-varían negativamente con una caída vertical en los primeros 40 años. Puede observarse que existe una caída exponencial conforme la literatura citada envejece y es evidente una tendencia en la que los altos valores de las frecuencias de las citas están asociados con la edad de la literatura más reciente, y los valores más bajos de las frecuencias de las referencias están asociados con las edades más antiguas de la literatura citada.

\section{Conclusiones}

La literatura revisada establece que un documento es obsoleto cuando deja de ser citado, es decir, cuando deja de ser usado por una comunidad académica como fuente de información para justificar, argumentar o contradecir las afirmaciones o hallazgos reportados por otros autores. Los resultados de esta investigación muestran que otras variables pueden estar influyendo sobre la obsolescencia y que generalmente esas variables no son mencionadas en la literatura dedicada a analizar el fenómeno conocido como "obsolescencia de la literatura". En el caso de la literatura sobre la Ley de Lotka, uno de esos factores es la lengua materna del autor del documento. El idioma de publicación es un factor que tiene un alto impacto sobre la obsolescencia de un documento. En la literatura publicada sobre la Ley de Lotka se verificó que un alto porcentaje de los documentos son publicados en inglés, pero esos documentos sólo citan otros documentos también publicados en inglés, mostrando un comportamiento considerado endogámico, por no decir etnocéntrico de parte de los autores que publican en inglés. Esta comprobación es sumamente desfavorable para aquellos que publican en otros idiomas pues sus publicaciones no serán citadas por esa comunidad llamada "internacional". Una comunidad "internacional" debería ser capaz de leer, entender y citar la producción de investigaciones por lo menos en dos o tres idiomas diferentes a los del contexto cultural del autor. No hacerlo implica un comportamiento endogámico y etnocéntrico. Ese comportamiento endogámico no se produce entre los autores que publican en idiomas diferentes al inglés. Estos autores siguen con atención no sólo lo que se produce en sus propios idiomas, sino también lo que se produce en inglés. Los casos de quienes publican en portugués y español ejemplifican este tipo de comportamiento.

El trazado de la distribución de la "nube de puntos" de la literatura no-acumulada sobre la Ley de Lotka publicada de 1926 hasta 2010 no 
muestra un patrón de obsolescencia definido sino una agrupación de los datos en tres grupos aleatorios, indicando ausencia del fenómeno "obsolescencia”. Sin embargo, la distribución de las citas acumuladas sincrónicamente según la edad de la literatura citada produce una caída de forma exponencial. Esta forma exponencial muestra una tasa de envejecimiento de $3.9 \%$ al año, relacionando una tasa media de envejecimiento a la edad de 17.4 años. Pero estos resultados parecen más un artificio de la acumulación de los datos que una real obsolescencia de la literatura. Esto es ejemplificado por los datos no agrupados que no muestran una caída obsolescente según la edad de la literatura citada.

\section{Bibliografía}

Abramescu, A. (1979), "Actuality and obsolescence of scientific literature", en Journal of the American Society for Information Science, vol. 30, núm. 5, septiembre, pp. 296-303.

Ahmed, Tanzila; Johnson, Ben; Oppenheim, Charles \& Peck, Catherine (2004), "Highly cited old papers and the reasons why they continue to be cited: Part II. The 1953 Watson and Crick article on the structure of DNA", en Scientometrics, vol. 61, núm. 2, pp. 147-156.

Baldauf, R. B. (1986), "Linguistic constrains on participation in psychology", en The Psychologist, vol. 41, pp. 220-240.

Bernal, J. D. (1958), "The transmission of scientific information”, en Proceedings of the International Conference on Scientific Information, Washington, D.C.: NAS, NRC, vol. 1, pp. 77-96.

Bourne, Charles P. (1962), "The world's journal literature: an estimate of volume, origin, language, field, indexing and abstracting", en American Documentation, vol. 13, núm. 2, abril, pp. 59-168.

- (1965), "Some user requirements stated qualitatively in terms of the 90 per cent library", en Allen Kent \& Orrin E. Taubee (eds.), Eletronic Information Handling, Washington, D.C.: Spartan Books, pp. 389-401.

Brookes, Bertram C. (1970a), "Obsolescence of special library periodicals: sampling errors and utility contours", en Journal of the American Society for Information Science, vol. 21, septiembre, pp. 320-329.

- (1970b), "The growth, utility and obsolescence of scientific periodical literature", en Journal of Documentation, vol. 26, núm. 4, diciembre, pp. 83-294.

_ (1972), "The aging of scientific literature", en Problems of Information Science: collection of papers, A. I Cherny (ed.), Moscow: International Federation of Documentation, Study Committee "Research on Theoretical Basis of Information", pp. 66-90. 
(1973), "Numerical methods of bibliographical análisis", en $L i$ brary Trends, vol. 22, núm. 1, julio, pp. 18-43.

Bronmo, Ole A (1978), "On the influence of availability on the use of Monographs in library criticism", en Tidskrift for Dokumentation, núm. 34, pp. 81-83.

Bulick, Stepehen et al. (1976), "Use of library materials in terms of age", en Journal of the American Society for Information Science, núm. 27, mayo/junio, pp. 75-178.

Burrell, Quentin L. (2002a), "Will this paper ever be cited?", en Journal of the American Society for Information Science, vol. 53, núm. 3, pp. 232-235.

- (2002b), "The nth-citation distribution and obsolescence", en Scientometrics, vol. 53, núm. 3, pp. 309-323.

- (2003a), "Age-specific rates and the Egghe-Rao function", Information Processing and Management, vol. 39, núm. 5, septiembre, pp. 761-770.

(2003b), "Predicting future citation behavior", en Journal of the American Society for Information Science, vol. 54, núm. 5, pp. 372-378.

Burton, R. E. \& Kebler, R. W. (1960), "The half-life of some scientific and technical literatures", en American Documentation, vol. 11, núm. 2, enero, pp. 18-22.

Chen, C. C. (1972), "The use patterns of Physics Journals in a Large Academic Research Library", en Journal of de American Society for Information Science, núm. 23, pp. 154-270.

Chen, Ye-Sho \& Leimkuhler, Ferdinand F. (1986), "A relationship between Lotka's law, Bradford's law and Zipf's law”, en Journal of the American Society for Information Science, vol. 37, núm. 5, septiembre, pp. 307-314.

Egghe, Leo (1994), "A theory of continuous rates and applications to the theory of growth and obsolescence rates", en Information Processing and Management, vol. 30, núm. 2, pp. 279-292.

( $2005 \mathrm{a})$, "The power of power laws and an interpretation of Lotkaian Informetric Systems as self-similar fractals", en Journal of the American Society for Information Science and Technology, vol. 56, núm. 7, pp. 669-675.

(2005b), "Relations Between the Continuous and the Discrete Lotka Power Function", en Journal of the American Society for Information Science and Technology, vol. 56, núm. 7, pp. 664-668.

— \& Ravichandra Rao I. K. (1992a), "Classification of growth models base on growth rates and its applications", en Scientometrics, vol. 25, núm. 1, pp. 5-46.

— \& - (1992b), "Citation age data and the obsolescence function: fits and explanations", en Information Processing E Management, vol. 28, núm. 2, pp. 201-217.

— \& - (2002), "Theory and experimentation on the most-recentreference distribution", en Scientometrics, vol. 53, núm. 3, pp. 371387. 
- \& Rousseau, Ronald (2000), "The influence of publication delays on the observed aging distribution of scientific literature", en Journal of the American Society for Information Science, vol. 51, núm. 2, enero, pp.158-165.

Ewing, Gordon J. (1966), "Citation of articles from volume 58 of the Journal of Physical Chemistry", en Journal of Chemical Documentation, vol. 6, núm. 4, noviembre, pp. 247-250.

Gapen, D. Kaye \& Milner, Sigrid P. (1981), "Obsolescence”, en Library Trends, vol. 30, núm. 1, verano, pp. 107-124.

Glänzel, Wolfgang (2004), "Towards a model for diachronous and synchronous citation analyses", en Scientometrics, vol. 60, núm. 3, pp. 511-522.

Gosnell, Charles F. (1943), The rate of obsolescence in college library book collections as determined by the analysis of three selected list of books for college libraries, Ph. D. Dissertation, New York: University of New York.

Gupta, B. M. (1998), "Growth and obsolescence of literature in theoretical population genetics”, en Scientometrics, vol. 42, núm. 3, pp. 335-347.

Gupta, Usha (1990), "Obsolescence of physics literature: exponential decrease of the density of citations to Physical Review articles with age", en Journal of the American Society for Information Science, vol. 41, núm. 4, junio, pp. 282-287.

Heisey, T. M. (1988), "Paradigm agreement and literature obsolescence: a comparative study in the literature of Dead Sea Scrolls", en Journal of Documentation, vol. 44, pp. 285-301.

Hubert, J. J. (1978), “A relationship between two forms of Bradford's law", en Journal of the American Society for Information Science, vol. 29, núm. 2, pp. 159-161.

Line, M. B. (1993), "Changes in the use of literature with time: obsolescence revisited”, en Library Trends, vol. 41, núm. 4, primavera, pp. 665-678.

- (1970a), "The 'half-line' of periodical literature: apparent and real obsolescente", en Journal of Documentation, vol. 26, núm. 1, marzo, pp. 46-54.

(1970b), "Does physics literature obsolesce? A study of variation of citation frequency with time for individual journal articles in physics", en British Lending Library Review, vol. 2, pp. 84-91.

_ \& Sandison, A. (1974), "Obsolescences and changes in the use of literature with time", en Journal of Documentation, núm. 30, pp. 284-350.

Longyear, R. M. (1977), “Article citations and 'obsolescence' in musicological journals", en Quarterly Journal of the Music Library Association, vol. 33, pp. 563-571.

Lotka, Alfred (1926), "The frequency distribution of scientific productivity", en Journal of the Washington Academy of Sciences, vol. 16, núm. 12, junio, pp. 317-323. 
McCain, Katherine W. (1987), "Citation patterns in the history of technology”, en Library and Information Science Research, vol. 9, pp. 41-59.

Miranda, Antonio Lisboa Carvalho de (1982), "Latin american periodicals in the field of library science: an analysis", en IFLA Conferencia Gral. (48: 1982: Montreal), Rio de Janeiro.

__ (1981), "Revistas especializadas brasileiras em biblioteconomia e ciencia da informação: com enfase na experiencia da ABDF", en Boletim ABDF: Nova Serie, Brasilia, octubre-diciembre, vol. 4, núm. 4, pp. 30-42.

Nicholls, Paul Travis (1986), "Empirical validation of Lotka's Law", en Information Processing and Management, vol. 22, núm. 5, pp. 417-419.

_ (1987), "Estimation of Zipf parameters", en Journal of the American Society for Information Science, vol. 38, núm. 6, pp. 443-445. Notes: Errata (1988): Journal of the American Society for Information Science, vol. 39, núm. 4, p. 287.

_ (1988), "Price's square root law: empirical validity and relation to Lotka's Law", en Information Processing and Management, vol. 24, núm. 4, pp. 469-477.

_ (1989), "Bibliometric modeling processes and the empirical validity of Lotka's Law", en Journal of the American Society for Information Science, vol. 40, núm. 6, pp. 379-385.

Pao, Miranda Lee (1986), "An empirical examination of Lotka’s law", en Journal of the American Society for Information Science, vol. 37, núm. 1, enero, pp. 26-33.

_ (1985), "Lotka's Law: a testing procedure", en Information Processing and Management, vol. 21, núm. 4, pp. 305-320.

Price, John Derek de Solla (1965), "Networks of scientific papers", en Science, vol.149, núm. 3683, pp. 510-515.

_ (1963), "Galton revisited", en Litle Science, Big Science, New York, N.Y.: Columbia University Press, pp. 33-61.

_ (1971), "The expansion of scientific knowledge", en Annals of the New York Academy of Sciences, New York, vol. 184, pp. 257259.

- (1976), "A general theory of bibliometric and other cumulative advantage processes", en Journal of the American Society for Information Science, vol. 27, núm. 5/6, pp. 292-306.

Ravichandra Rao, I. K. \& Meera, B. M. (1992), "Growth and obsolescence of literature: an empirical study", International Conference on Bibliometrics, Scientometrics, and Informetrics (1991, Bangalore, India), en Informetrics 91: selected papers from the third International Conference on Informetrics, 9-12 de agosto, Bangalore: Sarada Ranganathan Endowment for Library Science, pp. 377-394.

Referí de la REDC (Revista Española de Documentación Científica), comunicación enviada a Rubén Urbizagástegui el viernes 24 de junio del 2011. 
Rousseau, Ronald (1992), "Breakdown of the robustness property of Lotka's Law: the case of adjusted counts for multiauthorship attribution", en Journal of the American Society for Information Science, vol. 43, núm. 10, pp. 645-647.

_ (1993), "A table for estimating the exponent in Lotka's law", en Journal of Documentation, vol. 49, núm. 4, pp. 409-412.

Rousseau, Brendan \& Rousseau, Ronald (2000), "LOTKA: A program to fit a power law distribution to observed frequency data", en $C y$ bermetrics, vol. 4, núm. 1, pp. 1-6.

Sandison, A. (1971a), "The use of older literature and its obsolescence", en Journal of Documentation, vol. 27, núm. 3, pp. 184-199.

__ (1971b), "Library optimum", en Nature, núm. 234, pp. 368-379.

_ (1974), "Densities of use, and absence of obsolescence in Physic journal at MIT", en Journal of the American Society for Information Science, vol. 25, núm. 3, mayo-junio, pp. 172-182.

— (1975), "Patterns of citation densities by date of publication in Physical Review", en Journal of the American Society for Information Science, vol. 26, núm. 6, pp. 349-351.

Stinson, Eddie Ray (1981), Diachronous vs. synchronous study of obsolescence, Thesis (Ph. D.), University of Illinois at Urbana-Champaign.

_ \& Lancaster, F. W. (1987), "Diachronous vs. synchronous methods in the measurement of obsolescence by citation Studies", en Journal of Information Science, vol. 13, núm. 2, pp. 65-74.

Seymour, Carol A. (1972a), "Weeding the collection: a review of research on identifying obsolete stock: monographs (part 1)", en Libri, núm. 22, pp. 137-148.

_ (1972b), "Weeding the collection: a review of research on identifying obsolete stock: serials (part 2)”, en Libri, núm. 22, pp. 183 189.

Taylor, Colin R. (1976-1977), "A practical solution to weedding University Library collections", en Collection Management, núm. 1, otoño-invierno, pp. 27-45.

Urbizagástegui Alvarado, Rubén (2009), "Crescimento da literatura e dos autores sobre a Lei de Lotka”, en Ciencia da Informação, Brasilia, vol. 38, núm. 3, septiembre-diciembre, pp. 111-129.

Van Raan, Anthony F. J. (2000), "On growth, ageing, and fractal differentiation of science”, en Scientometrics, vol. 47, núm. 2, pp. 347362.

Wallace, Danny P. (1986), "The relationship between journal productivity and obsolescence", en Journal of the American Society for Information Science, vol. 37, núm. 3, mayo, pp. 136-145.

Watson, James D. \& Crick, Francis (1953), Molecular structure of nucleic acids: a structure for deoxyribonucleic acid, St. Albans: Fisher, Knight, 1953. 
Yablonsky, A. I. (1980), "On fundamental regularities of the distribution of scientific productivity”, en Scientometrics, vol. 2, núm. 1, pp.3-34.

Zafrunnisha, N. \& Reedy, V. Pulla (2010), "Citations in Psychology PhD Theses: An Obsolescence Study", en Library Pbilosophy and Practice, vol. 7, núm. 27, julio, pp. 1-8.

$\infty$ 